\title{
A Flexible Error Correction Scheme for IEEE 802.15.4-based Industrial Wireless Sensor Networks
}

\author{
Kan Yu*, Filip Barać ${ }^{\dagger}$, Mikael Gidlund ${ }^{\ddagger}$, Johan Åkerberg ${ }^{\ddagger}$ and Mats Björkman* \\ *Mälardalen University, Sweden \\ ${ }^{\dagger}$ Mid Sweden University, Sweden \\ $\ddagger$ ABB AB, Corporate Research, Sweden
}

\begin{abstract}
Noise and interference make a substantial impact on wireless transmissions in industrial environments, resulting in frequent erroneous packet deliveries. Existing industrial communication standards adopt the IEEE 802.15.4 specification, which provides no means to correct the detected errors. We propose an IEEE 802.15.4-compliant Forward Error Correction-based approach that can be easily retrofitted into the standard without the need for any kind of interaction with chip manufacturers or standardization bodies. We evaluate the approach on link- and network-level scenarios. Improvement of reliability by using FEC can yield multiple benefits: a reduced number of retransmissions, and lower average latency, to name a few. With respect to the uncoded system, the proposed solution provides identical coding gain as the traditional FEC method, at a significantly lower computational load of decoding.
\end{abstract}

\section{INTRODUCTION}

The penetration rate of Wireless Sensor Networks (WSNs) in Process Automation continues to increase. Industrial Wireless Sensor Network (IWSN) technologies have found their place in industrial control systems due to the flexibility and cost reduction that they offer. However, a number of physical factors threatens to compromise the fulfillment of strict realtime and reliability requirements in IWSNs [1]. Two methods of improving communication reliability are Automatic Repeat Request and Forward Error Correction.

Automatic Repeat Request (ARQ) is based on packet retransmissions with an inherent drawback of increased latency. This is in direct conflict with time-critical requirements of industrial applications. The empirical results from [2] revealed that excessive retransmissions may lead to more serious consequences, such as network congestion and complete halt of the observed industrial process.

In Forward Error Correction (FEC), reliability is improved by introducing redundant information in the original data. The literature on FEC coding in WSNs is plentiful. However, to the best of our knowledge, the works in [3] and [4] are the only works providing a packet-level insight of coding in WSN, whereas this work is an enhancement of [4]. The authors of [3] introduce a MAC layer FEC scheme with Reed-Solomon $(15,7)$ code, but this implementation violates the IEEE 802.15.4 format by reducing the control fields below the minimum dimensions prescribed by the standard. A lightweight XOR-based FEC algorithm in [5] fragmentizes and sends the original data over multiple paths. XOR-based checksums of data fragments are sent in separate packets.
Such an approach would be highly inefficient for small IWSN packets due to header and traffic overhead. Furthermore, the checksum packet loss stultifies the entire transmission, since the integrity of data fragments could not be checked in the sink. A vast majority of FEC-related work for WSNs focuses on energy efficiency of the coding. In [6], relationships are set between the energy efficiency of FEC scheme, communication distance and packet size. Cross-layer approaches yielding energy-efficient designs of FEC schemes are proposed in [7] and [8]. The authors of [9] investigate the performance of adaptive FEC schemes, without considering the IEEE 802.15.4 standard. Energy consumption is not considered in this paper.

With respect to the state-of-the-art works, we propose an IEEE 802.15.4-compatible FEC scheme on the MAC layer, that can be retrofitted and deployed immediately. We argue that FEC on the MAC layer is more appropriate than on the Physical (PHY) layer. Two properties of the proposed solution are particularly important for its applicability in practice. Firstly, the packet format prescribed by the IEEE 802.15.4 specification must be maintained, for backward compatibility reasons. Our solution incorporates FEC coding on the MAC layer without disrupting the packet format defined by the specification. This not only provides a smooth integration of FEC codes into the standard, but also allows nodes that do not employ FEC coding to participate in the network. Secondly, the FEC codes employed must have low complexity, with respect to the computational and memory resources [4]. The scheme is flexible in the sense that the number of decoding actions on a packet at an intermediate node before being forwarded ranges from a simple correctness check, in the case of an uncorrupted packet, to full decoding and error correction in the worst case. Our solution also liberates the network from carrying irreparable packets. We show that the proposed FEC scheme exhibits a significantly better performance than the uncoded approach. Compared with traditional FEC scheme, a remarkable reduction of decoding operations is also achieved by our solution.

Section II of this paper briefly describes the basic concepts of the IEEE 802.15.4 standard and FEC coding. In Section III we introduce the proposed FEC solution and analyze the theoretical error performance. Section IV describes the experimental setup and simulation scenarios, followed by the discussion and analysis of the results in Section V. Section VI concludes the article. 


\section{Preliminaries}

This section gives basic details of IEEE 802.15.4 specification, the de facto standard for WSN, as well as the principle of FEC coding.

\section{A. IEEE 802.15.4 standard}

The IEEE 802.15.4 standard [10] specifies both Physical (PHY) and MAC layers. PHY layer uses Direct Sequence Spread Spectrum (DSSS), in order to increase the robustness of the signal in the heavily saturated Industrial, Scientific and Medical (ISM) band, where both IEEE $802.11 \mathrm{~b} / \mathrm{g}$ and Bluetooth signals are present. The standard also allows operation in 868/915 MHz band. In MAC layer, Carrier Sense Multiple Access with Collision Avoidance (CSMA/CA) mechanism prevents packet collisions, and ARQ mechanism improves the reliability of communication, but at a price of increased latency.

\section{B. Forward Error Correction}

FEC coding introduces redundancy into the transmitted data, in order to preempt the problem of potential packet corruption and recover the original data. In this work we employ ReedSolomon [11] (RS) block codes with hard decision decoding. Provided the original message length is $k$ symbols a FEC encoder is adding $n-k$ redundant symbols to form an $n$ symbol codeword i.e. block. A newly formed codeword can tolerate a certain number of bit errors, while still being fully retrievable in the FEC decoder.

\section{Proposed Enhanced FEC SCHEME For IEEE} 802.15 .4

In this section we describe our proposed FEC scheme for IEEE 802.15.4 and analyze the error performance with and without the MAC-level FEC protection.

\section{A. Applying FEC Codes at the MAC layer}

In most of the cases, due to the lack of access to the "silicon", applying FEC on the MAC layer is more appropriate and flexible than on the Physical layer, especially for software implementations. Theoretically, protecting all layers, including PHY, may increase reliability. However, even if applying FEC on the PHY layer is feasible, it means involving the packet preamble and PHY header in the FEC processing. The preamble is used for synchronization and PHY header carries the information about packet length. Coding, for instance, the preamble, which is the largest field in the PHY, will not provide higher gain, since the corruption of preamble leads to packet loss or drastically increases the bit-stuffing process in order to guarantee integrity and eliminate out-of-sync packet receptions.

The choice of FEC code in this work is based on the conclusions from [4]. Systematic FEC codes with decent speed constrained by the timing requirement from the standard are appropriate for our proposed FEC scheme. The authors have shown that Reed-Solomon $\operatorname{RS}(15,11)$ block code [11] is suitable for IEEE 802.15.4-based IWSNs. Balakrishnan et al. [12]

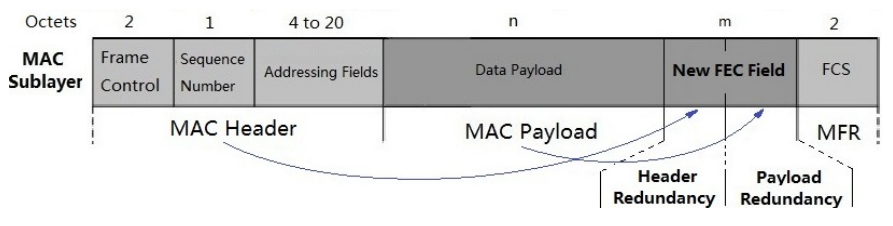

Fig. 1. Proposed FEC Encoded Data Frame structure

claim that that binary $\mathrm{BCH}$ codes with Application-Specific Integrated Circuit (ASIC) implementation are most suitable for WSNs. However, in many cases, software implementation is still more flexible at a lower cost than the hardware-based approach. Due to the reasons previously stated, we apply FEC codes in MAC layer instead of PHY. Hence, in order to make packet detection possible, IEEE 802.15.4 preamble and PHY header are not encoded.

In this paper, we propose a flexible FEC scheme applied in MAC layer of IEEE 802.15.4 for multi-hop IWSNs. Our solution places the redundancy obtained by coding the header and coding the payload into the field denoted as New FEC Field at the end of the MAC payload of the original data frame, as shown in Figure 1. The first part saves the FEC redundant data generated from the MAC header and the second part saves the data from the payload, which has slight implications on the encoding scheme. The complete MAC layer packet is split into three parts: the header, payload and the checksum field. Both MAC header and payload are further divided into symbol groups of size corresponding to FEC code message length. Zero padding is applied to the last group if its length is less than the FEC message length. Each block is encoded with systematic FEC codes, except for the Frame Check Sequence (FCS) field. Finally, the added redundancy from each encoded group is stored into the corresponding place inside the new FEC field following the payload. A new flag in the MAC header indicates whether FEC coding is used or not.

The decoding procedure of our FEC scheme is an improvement of [4]. In this work, the encoded MAC header and payload are rebuilt and processed separately by the decoder. An intermediate node in multihop communication is able to check the packet by processing the FCS checksum. If no bit error is found, the node can determine, without decoding the packet, whether it is the final destination, an intermediate node, or it should just ignore the packet. This is possible because the PHY header is left uncoded and MAC header in a coded packet is still visible to the node. By enabling the nodes to directly decide whether to forward or discard the packet, the entire decoding procedure is avoided, and network is spared from carrying potentially irretrievable packets. If FCS check indicates the presence of errors, the packet should be decoded and processed.

Another important advantage of this scheme is the compatibility with the original standard. Since the syntax of the encoded packet is preserved, a sensor without FEC scheme implemented is still able to process the encoded packet normally. This fact has a major significance for multi-hop networks, since the lack of FEC scheme implemented on sensor nodes 
will not affect packet forwarding. Further optimization of our previously proposed FEC scheme aims at simplifying the required number of operations taken by the routing sensor nodes in a multi-hop network. Since the MAC header and payload are encoded separately, if a corrupted packet only contains errors in the MAC header, the routing node, which has FEC scheme implemented, only needs to recover the header by error-correction, instead of decoding the whole packet. The actions taken by routing nodes to forward packets are summarized in Algorithm 1. This optimized FEC scheme reduces the workload of routing nodes and makes the procedure more flexible. It is noticeable from the algorithm that routing nodes shall guarantee the correctness of packets. Irreversibly corrupted packets shall not be forwarded, thus saving network resources and bandwidth and reducing the processing load.

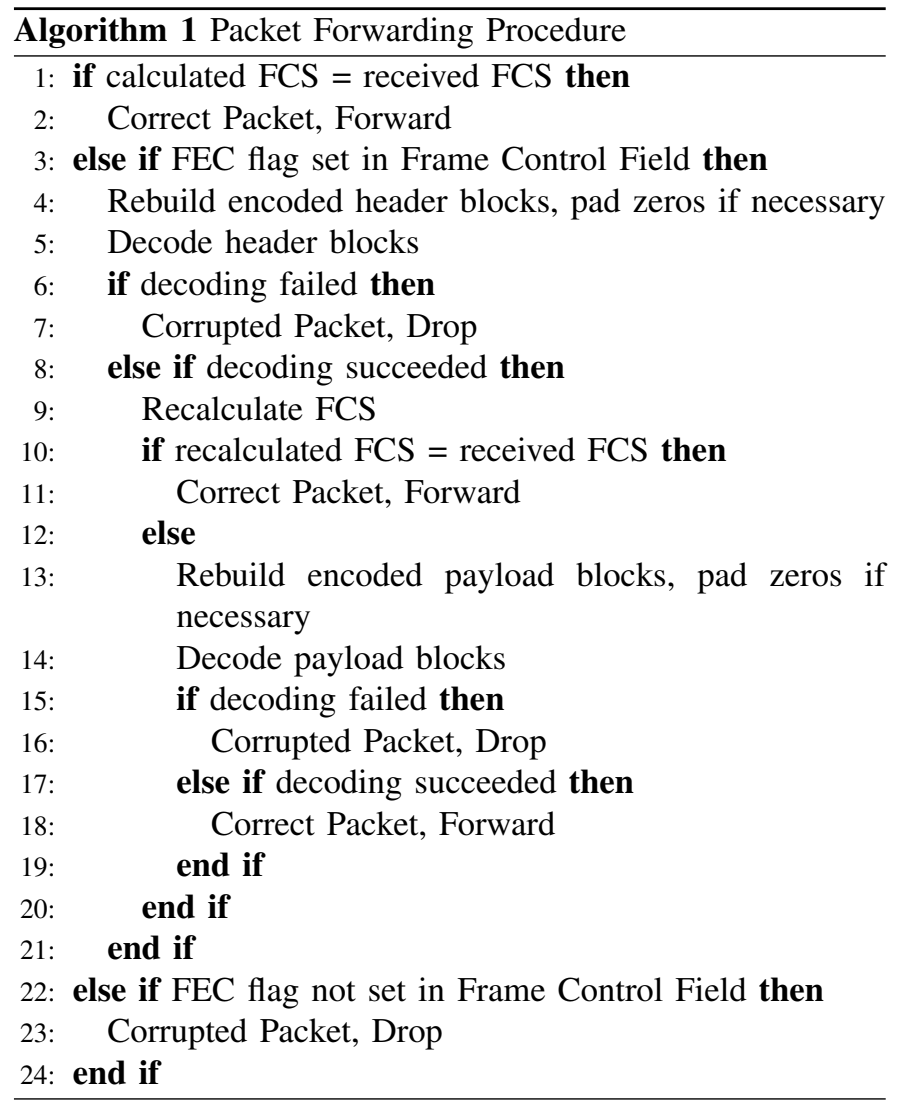

By our proposed solution, the implementation complexity is increased, as well as the computational load of transmitter nodes. In addition, compared with traditional FEC methods, our solution does not provide an increase in overall Packet Delivery Ratio (PDR). However, the compatibility with IEEE 802.15.4 and the flexibility for multihop networks are achieved, as elaborated above. This can also be observed from the proposed packet forwarding algorithm, whose properties make it more suitable for multihop IEEE 802.15.4-based IWSNs than traditional FEC schemes.

\section{B. Error Performance Analysis}

In this subsection, we provide an error performance analysis of both uncoded and coded transmissions, based on the proposed FEC scheme. We assume that the effect of the interferers on the individual packet level is similar to Additive White Gaussian Noise (AWGN) with power spectral density $N_{0} / 2$ in the same bandwidth, since it is believed that the analysis based on AWGN model leads to the similar result as the one based on a more practical channel model.

IWSNs usually operate in $2.4 \mathrm{GHz}$ band with Offset Quadrature Phase Shift Keying (OQPSK) modulation scheme. For OQPSK modulation with DSSS, the Bit Error Rate (BER) without considering the phase noise is given by [13]:

$$
P_{b}=\frac{M_{s} / 2}{M_{s}-1} \sum_{k=1}^{M_{s}-1} \frac{(-1)^{k+1}}{k+1}\left(\begin{array}{c}
M_{s}-1 \\
k
\end{array}\right) e^{-\frac{k}{1+k} \frac{K_{b} E_{b}}{N_{0}}}
$$

where $K_{b}=\log _{2} M_{s}$ denotes the number of bits per symbol, $M_{s}=16$ is the size of the signal constellation, and $E_{b}$ represents the energy per bit.

For an uncoded system, provided the whole packet length, including the PHY layer header, is $l$ bits, the Packet Error Rate (PER) $P_{e}$ is [13]:

$$
P_{e}\left(P_{b}, l\right)=1-\left(1-P_{b}\right)^{l}
$$

For coded transmissions, we evaluate the performance of our proposed FEC scheme with $\mathrm{RS}(15,11)$ code. For $R S(n, k)$ codes, $k$ indicates the original data length and $n$ is the length of the codeword. The error correction capability is $t=(n-k) / 2$. Provided that the block length of RS is $n$ bits, the probability that a codeword is wrongly decoded is given by [13]:

$$
P_{r}\left(P_{e}^{s y m}, n\right)=\sum_{i=t+1}^{n}\left(\begin{array}{c}
n \\
i
\end{array}\right)\left(P_{e}^{s y m}\right)^{i}\left(1-P_{e}^{s y m}\right)^{n-i}
$$

where $P_{e}^{s y m}$ is the symbol error rate obtained from (2). The symbol length depends on the RS code used.

In our proposed FEC scheme, there are three sections in a packet that are out of the FEC protection range: the synchronization header, PHY header and MAC layer FCS field. Although FCS field is not FEC-encoded, its corruption will not lead to the failure of packet decoding, as it was motivated in the forwarding and decoding algorithms. This does not hold for corruption of the synchronization header and PHY header, in case of which our FEC scheme cannot contribute to the performance improvement.

For operation in the $2.4 \mathrm{GHz}$ frequency band, as required by the IEEE 802.15 .4 standard, the synchronization header consists of the Preamble field of 4 bytes and start-of-frame delimiter of 1 byte, as well as 1-byte PHY header. Thus, the total length of these three components is 48 bits. We assume that the MAC layer header is $h$ bits long, so the payload is $l-h-48-2 \times 8=l-h-64$ bits long. According to our scheme, a one-bit FEC flag is always checked before decoding, so the corruption of the flag will also result in the failure of 


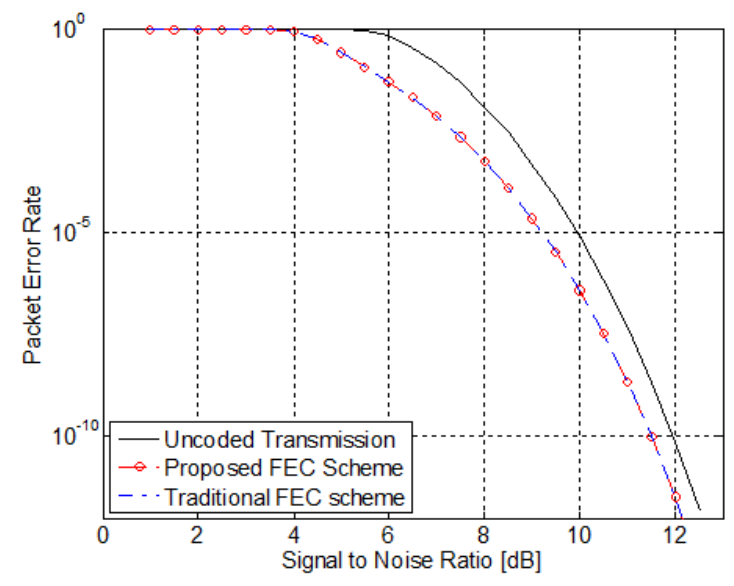

Fig. 2. Theoretical PER of the proposed solution, traditional RS(15,11) scheme and uncoded transmissions

packet reception. Therefore, the PER of our proposed FEC scheme $P_{e}^{\prime}$ can be written as:

$$
\begin{aligned}
P_{e}^{\prime}\left(P_{b}, P_{r}\right)= & 1-\left(\left(1-P_{b}\right)^{48}\left(1-P_{b}\right)\right. \\
& \left.\times\left(1-P_{r}\right)^{\left\lceil\frac{h}{m k}\right\rceil}\left(1-P_{r}\right)^{\left\lceil\frac{(l-h-64)}{m k}\right\rceil}\right) \\
= & 1-\left(\left(1-P_{b}\right)^{49}\left(1-P_{r}\right)^{\left(\left\lceil\frac{h}{m k}\right\rceil+\left\lceil\frac{(l-h-64)}{m k}\right\rceil\right)}\right)
\end{aligned}
$$

where $P_{r}$ is obtained from (3) and $m$ is the number of bits in one RS symbol.

Figure 2 shows the dependence of PER on received SNR for the proposed solution, traditional $\mathrm{RS}(15,11)$ coding scheme, as well as uncoded transmissions. The gain of our solution with respect to the uncoded system is $1 \mathrm{~dB}$ at the PER of $10^{-3}$. The proposed solution matches the performance of the traditional FEC approach in the entire observed SNR range. The traditional approach refers to directly applying FEC code to the original data without additional operations, contrary to the proposed solution, which was described above. Although our solution does not achieve lower PER than the traditional approach, it aims for providing compatibility to the existing standard and reducing the computational load of sensor nodes.

The code rate indicates the size of overhead introduced in the transmissions, which affects transmission efficiency. The code rate of our proposed scheme $R^{\prime}$ is:

$$
R^{\prime}=\frac{l R}{\left(\left\lceil\frac{h}{m k}\right\rceil+\left\lceil\frac{l-h-64}{m k}\right\rceil\right)+64 R}
$$

where $R=k / n$ is the original code rate from RS code.

\section{EXPERIMENTAL SETUP}

This section presents the particularities of executed simulations.

\section{A. Simulation Environment and Measurables of Interest}

The experiments are executed in QualNet discrete event simulator [14] in point-to-point and mesh network topologies. The performance of our approach is compared with the performance of uncoded IEEE 802.15.4 communication, as well as the traditional RS scheme. Since the original IEEE 802.15.4 standard employs error detection, rather than error correction, the number of corrupted packets for uncoded transmission equals the number of irreparably corrupted ones. The simulation parameters are presented in Table I.

TABLE I

THE SIMULATION PARAMETERS

\begin{tabular}{cc}
\hline Parameter & Description \\
\hline Application layer & CBR application \\
Routing protocol & ZRP \\
MAC layer & IEEE 802.15 .4 \\
Physical layer & IEEE 802.15 .4 at $2.4 \mathrm{GHz}$ with O-QPSK \\
Fading Model & Rayleigh fading \\
Payload size & $74 / 119$ bytes@ APPL/MAC layer \\
Bit rate & $250 \mathrm{kbps}$ \\
Receiver Sensitivity & $-85 \mathrm{dBm}$ \\
\hline
\end{tabular}

Real-world IWSNs often operate in non-line-of-sight conditions, which can be well represented by the Rayleigh fading model. The signal subject to Rayleigh fading exhibits fast variations in amplitude and phase shifts at the receiver, mainly due to multipath propagation and relative motion of transmitter, receiver and surrounding objects, a notable trait of typical IWSN settings. This is the main motivation for including Rayleigh fading into the experiments.

\section{B. Packet Reception Model}

An IEEE 802.15.4 packet starts with six bytes of preamble, packet delimiter and PHY layer header. As previously explained, this part of the packet is left uncoded, and our proposed solution is to place the $\mathrm{RS}(15,11)$ code redundancy at the end of the packet. An irrepairable packet error occurs in the following cases:

- Bit errors in preamble and/or PHY header lead to packet loss, irrespective of whether the rest of the packet can be recovered or not.

- The number of corrupted bits exceeds the error correcting capability of the code. A symbol is considered corrupted if at least one of its bits is corrupted. For $\operatorname{RS}(15,11)$ code, two corrupted symbols (bytes) must be at least five symbols apart, in order for the packet to be decoded.

\section{Simulation Scenarios}

The performance of our approach is compared to the performance of uncoded transmissions and the traditional $\operatorname{RS}(15,11)$ FEC scheme in two scenarios. The measurements of interest in both scenarios are PDR and the number of decoding function calls. Communication in both scenarios is subject to Rayleigh fading. 


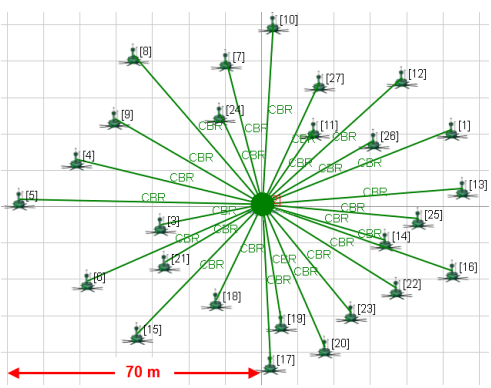

(a) Topology 1

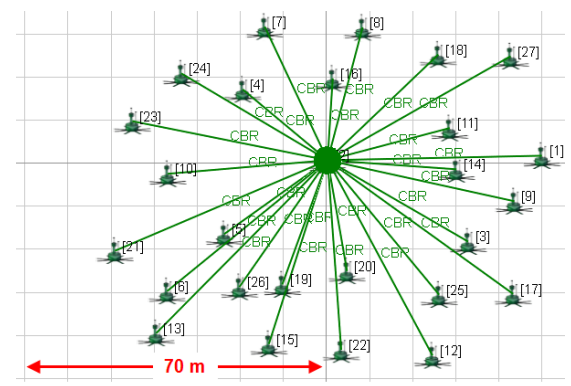

(b) Topology 2

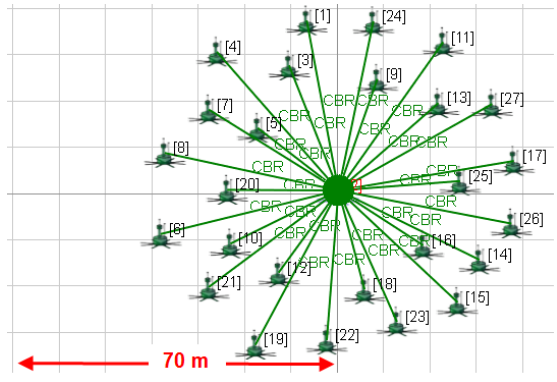

(c) Topology 3

Fig. 3. The three network topologies in Scenario II

1) Scenario I: According to the theory, transmitter-receiver separation is inversely proportional to PDR. The first scenario includes two sensor nodes at a distance of 75 meters, which is $75 \%$ of node's transmission range in the propagation model of the simulator, for the given transmit power. The sensor refresh rate is $500 \mathrm{~ms}$.

2) Scenario II: This scenario includes three random mesh topologies of 26 sensor nodes and a centrally located sink. All sensor nodes are instructed to report measurements with a refresh rate of $1 \mathrm{sec}$ and the topologies are shown in Figure 3 .

\section{Evaluation RESUlts AND Discussion}

This section presents and discusses the results obtained in the scenarios.

\section{A. Scenario I}

Figure 4 shows the PDRs and the number of decoding function calls for a communication between two IEEE 802.15.4 sensor nodes under Rayleigh fading. Compared to uncoded transmissions, both the proposed coding scheme and traditional FEC scheme contributes to a relative increase in PDR of $21 \%$. The receiver is able to retrieve a number of corrupted packets, provided none of the cases explained in Subsection IV-B occurs. In case of uncoded communication, all packet errors are irreparable, since only error detection is applied. Consequently, there is a significant difference in the number of successfully decoded packets in favor of FECcoded system. Considering the error correction capability of $\mathrm{RS}(15,11)$, this performance gain is within the expected range. The justification for similar PDR performance of the proposed scheme and traditional RS scheme can be found in Figure 2.

Although our proposed scheme provides a similar PDR performance to the traditional one, Figure 4 shows one of the benefits of our solution: the number of decoding function calls of sensor nodes is more than halved. The traditional scheme decodes the entire packet, regardless of whether errors have occurred in the MAC header or in the payload, or not at all. On the other hand, by our proposed solution, decoding function is called only when necessary, so the number of calls is reduced to a great extent.

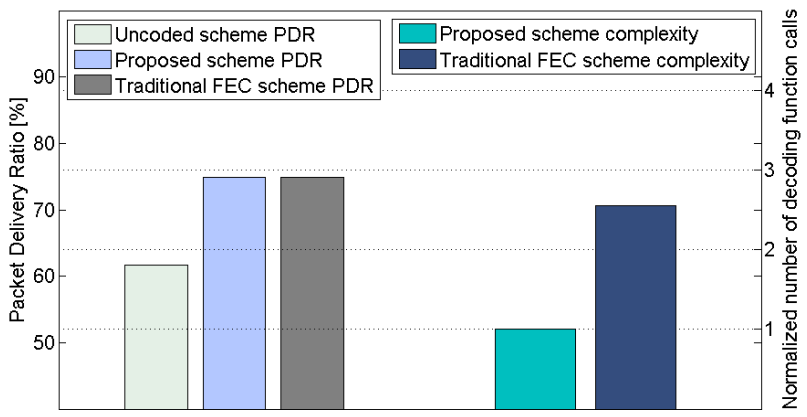

Fig. 4. Scenario I: PDR and the normalized number of decoding function calls in a Rayleigh fading channel

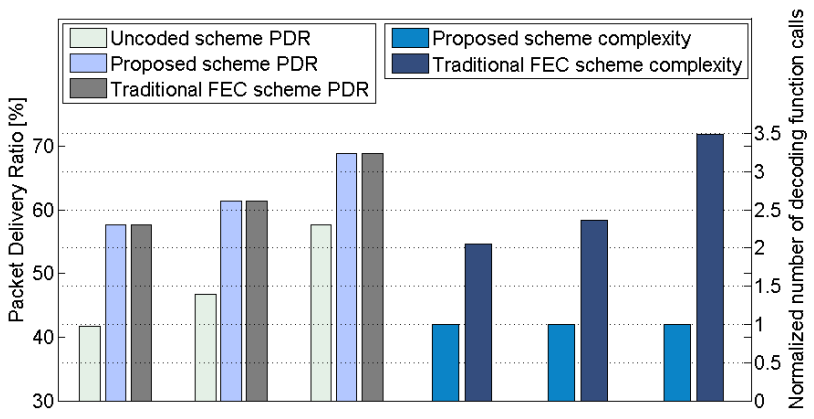

Fig. 5. Scenario II: PDR and the normalized number of decoding function calls in a Rayleigh fading channel

\section{B. Scenario II}

This experiment is an assessment of the proposed approach on three mesh topologies, shown in Figure 3. Each topology comprises a single sink and 26 randomly placed nodes, reporting their measurements to the sink every 1 second over a Rayleigh fading channel. The obtained PDRs the number of decoding function calls for three different network topologies are presented in Figure 5.

Compared to the uncoded scheme, the proposed solution in all three topologies shows a significant improvement in PDR. Namely, the absolute improvements in PDR are between 
$11 \%$ and $15 \%$, compared to the uncoded system. In terms of relative improvement, the communication reliability is boosted by $19 \%-38 \%$. Since the results from three different network topologies exhibit the same trend, it follows that a significant improvement of transmission reliability can be generally achieved by our proposed scheme. The variation in PDR between the topologies is the consequence of different nodesink separations. The average distance between the nodes and the sink in Topology 1 is the longest, whereas in Topology 3 it is the shortest. Accordingly, the overall PDR from Topology 1 is lower than in Topology 2 and 3. The transmission distance is highly correlated with the received SNR, and, as shown in Figure 2, our solution becomes fruitful for SNRs above $4 \mathrm{~dB}$.

Similarly to the results from Scenario I, the proposed solution provides almost identical PDR performance as the traditional RS $(15,11)$ scheme. However, in mesh IWSNs, the processing load of decoding in both intermediate and end nodes is significant, due to large traffic intensity. Different from the traditional FEC scheme, our proposed solution decouples the MAC header and payload decoding. Because of intermediate nodes in a multihop network, the MAC header is of a vital importance for packet forwarding and shall be always checked first. In case the errors have occurred solely in the MAC header, the proposed scheme shall only decode the header and it shall proceed to payload decoding only if the payload is corrupted, as well. Figure 5 shows the number of times the decoding function was called, for the traditional and proposed FEC scheme. For all three topologies, the number of decoding function calls is normalized by the number of times the decoding function was called by the proposed solution. It is notable from the results that the number of decoding function calls in Topology 1 is more than halved, compared to traditional RS $(15,11)$ decoding. In Topology 3, computational savings are even more drastic - the traditional FEC scheme called the decoding function 3.5 times more than the proposed scheme.

\section{CONCLUSIONS AND Future WORK}

In this work, we propose a MAC layer Forward Error Correction scheme for IEEE 802.15.4-based WSN. Any systematic block code with short execution time according to the standard can be applied in our proposed scheme and in this work $\mathrm{RS}(15,11)$ code is chosen. Since data integrity is checked on-the-fly, the transmission of erroneous packets is prevented and bandwidth is gained. The solution allows the nodes not running FEC coding to participate in the network, as well. PDR was boosted by $21 \%$ and up to $56 \%$ in the point-to-point and mesh scenarios, compared to uncoded transmissions. The increase in reliability will also cause the number of retransmissions to decline, which will translate to lower average packet latency. Our scheme provides identical PDR performance to the traditional RS $(15,11)$ scheme, but the number of decoding function calls is significantly reduced. This is made possible by decoupling of header and payload decoding and the reduction was observed in the experiments was 2-3.5 times.
The most notable advantage of our solution over the traditional FEC scheme is the full compatibility with the IEEE 802.15.4 specification and its implementation by no means violates the standard. Since our solution is applied on the MAC layer with software implementation, it does not require any hardware support for executing FEC nor interaction with the radio chip manufacturers. Therefore, as opposed to the traditional FEC scheme, our proposed solution is already deployable on existing platforms today.

Future research in this area is to scrutinize more suitable FEC code parameters and optimize the IWSN FEC code implementations for higher efficiency.

\section{REFERENCES}

[1] J. Åkerberg, M. Gidlund, and M. Björkman, "Future research challenges in wireless sensor and actuator networks targeting industrial automation," in IEEE 9th International Conference on Industrial Informatics (INDIN'11), July 2011.

[2] J. Åkerberg, M. Gidlund, F. Reichenbach, and M. Björkman, "Measurements on an industrial wireless hart network supporting profisafe: A case study," in IEEE Conference on Emerging Technologies and Factory Automation (ETFA'11), Sept. 2011.

[3] E. Sanchez, F. Gandino, B. Montrucchio, and M. Rebaudengo, "Increasing effective radiated power in wireless sensor networks with channel coding techniques," in Electromagnetics in Advanced Applications, 2007. ICEAA 2007. International Conference on, sept. 2007, pp. 403 -406 .

[4] K. Yu, J. Åkerberg, M. Gidlund, and M. Björkman, "Reliable and low latency transmission in industrial wireless sensor networks," in The First International Workshop on Wireless Networked Control Systems (WNCS), Sept. 2011, pp. 1-6.

[5] Z. Xiong, Z. Yang, W. Liu, and Z. Feng, "A lightweight fec algorithm for fault tolerant routing in wireless sensor networks," in Wireless Communications, Networking and Mobile Computing, 2006. WiCOM 2006.International Conference on, Sept. 2006, pp. $1-4$.

[6] Y. Sankarasubramaniam, I. Akyildiz, and S. McLaughlin, "Energy efficiency based packet size optimization in wireless sensor networks," in Sensor Network Protocols and Applications, 2003. Proceedings of the First IEEE. 2003 IEEE International Workshop on, 2003, pp. 1 - 8.

[7] H. Karvonen and C. Pomalaza-Raez, "A cross layer design of coding and awake/sleep periods in wsns," in Personal, Indoor and Mobile Radio Communications, 2006 IEEE 17th International Symposium on, Sept. 2006, pp. $1-5$.

[8] M. Vuran and I. Akyildiz, "Error control in wireless sensor networks: A cross layer analysis," Networking, IEEE/ACM Transactions on, vol. 17 no. 4, pp. $1186-1199$, Aug. 2009.

[9] Z. Liankuan, X. Deqin, T. Yi, and Z. Yang, "Adaptive error control in wireless sensor networks," in Wireless Sensor Network, 2010. IET-WSN. IET International Conference on, Nov. 2010, pp. $360-366$.

[10] "Ieee 802.15.4 standard: Wireless medium access control (mac) and physical layer (phy) specifications for low-rate wireless personal area networks (wpans)," pp. 1-323, 2006.

[11] I. S. Reed and G. Solomon, "Polynomial Codes Over Certain Finite Fields," Journal of the Society for Industrial and Applied Mathematics, vol. 8, no. 2, pp. 300-304, 1960

[12] G. Balakrishnan, M. Yang, Y. Jiang, and Y. Kim, "Performance analysis of error control codes for wireless sensor networks," in Information Technology, 2007. ITNG '07. Fourth International Conference on, April 2007, pp. $876-879$.

[13] J. Proakis and M. Salehi, Digital communications, 5th ed. McGrawHill, Sept. 2007.

[14] (2010) Scalable network technologies, http://www.scalablenetworks.com. 